\title{
Die qualitative Alkaloidzusammensetzung der Lupine im Lichte chromatographischer Untersuchungen
}

M. WIEWIÓROWSKI und M. D. BRATEK

\section{DIE VORAUSSETZUNGEN DER ARBEIT}

Eine ausführliche Zusammenfassung der bisherigen Errungenschaften auf dem Gebiete der Chemie und Physiologie der Lupinenalkaloide finden wir in den Arbeiten von T. A. Hen ry (1950), F. Galin o v sky (1952) und N. J. L e o n a r d (1953).

In Folge der bisherigen Arbeiten wurden aus verschiedenen wilden und anbaufähigen Lupinenarten ca. 17 Alkaloide isoliert und ihre Eigenschaften beschrieben. Die Konstitution einiger Alkaloide wurde festgestellt und durch Synthese bewiesen. In den anbaufähigen und in Polen
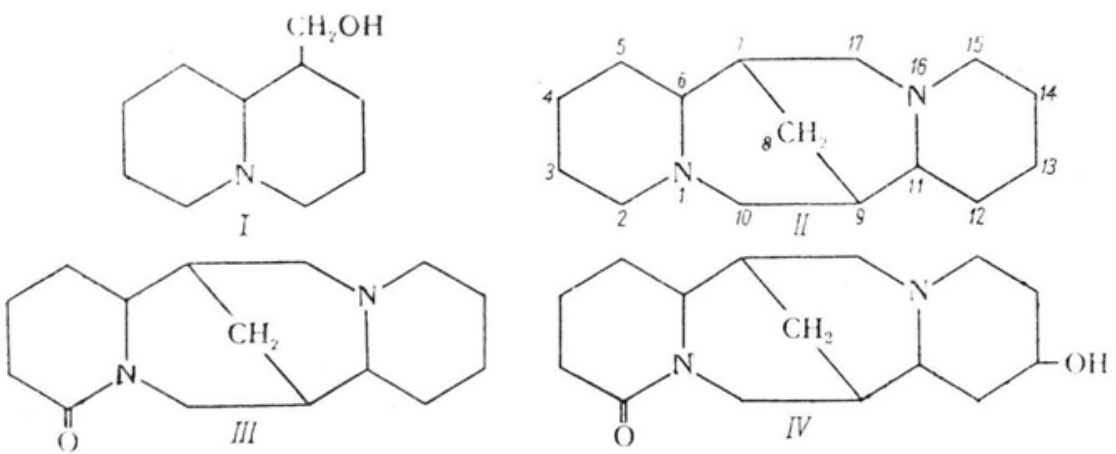

Abb. 1. I - Lupinin, II - Spartein, III - Lupanin, IV - Hydroxylupanin.

angebauten Lupinenarten wurden bisher 4 Alkaloide festgestellt. In der gelben Lupine wurde Lupinin (I) und Spartein (II) und in der weissen und schmalblättrigen - Lupanin (III) und Hydroxylupanin (IV) nachgewiesen.

Die erste Voraussetzung unserer Arbeit war eine Untersuchung, ob in den genannten Lupinenarten ausschliesslich nur die 4 Alkaloide anwe- 
send sind - und weiter, ob während der von S. B a r b a c ki (1952) geleiteten Züchtungsarbeiten, die eine Herabsetzung des Alkaloidgehaltes in den Lupinen zum Ziele haben, - sich nur die Menge der 4 Alkaloide ändert, oder ob sich etwa auch die qualitative Alkaloidzusammensetzung ändert.

Wir nahmen uns vor, die gesetzte Aufgabe mit Hilfe der chromatographischen Analyse zu lösen. Die chromatographische Methode wählten wir deshalb, weil uns keine Arbeit bekannt ist, die die chromatographische Analyse der Lupinenalkaloide betrifft, - und auch deshalb, weil uns die bisherigen Bestimmungsmethoden und Trennungsverfahren der Lupinenalkaloide zu wenig genau und zu brutal (Destillation bei verringertem Druck und einer Temperatur von $180^{\circ}$ bis $240^{\circ} \mathrm{C}$ ) vorkamen.

DIE ANWENDUNG DER PAPIERCHROMATOGRAPHIE ZUR QUALITATIVEN ANALYSE DER LUPINEN-ALKALOIDE

Anfangs führten wir unsere Versuche in Anlehnung an die Arbeiten von R. Munier, M. M a cheboeuf $(1949,1952)$ und Mitarbeitern aus, die in den Jahren 1949 bis 1952 veröffentlicht wurden und die $\mathrm{Pa}-$ pierchromatographie der Alkaloide und anderer biologischer Basen behandeln. Unsere ersten Versuche waren grossen Schwierigkeiten ausgesetzt, die hauptsächlich darauf beruhten, dass wir nicht über reine Präparate der in der Lupine vorkommenden Alkaloide verfügten. Wir waren lediglich im Besitz des leicht zugänglichen Sparteinsulfats und mit Hilfe dieses Salzes, und besonders mit Hilfe von Rohextrakten der aus der schmalblättrigen Lupine abgesonderten Alkaloide - arbeiteten wir an der Auswahl der Technik, der Lösungsmittelgemische und Entwicklungsreagentien.

\section{A. DIE CHROMATOGRAPHISCHE TECHNIK}

Aus der Vielzahl der chromatographischen Arbeitsmethoden wandten wir das für unsere Zwecke am besten geeignete Verfahren an. Als solches zeigte sich die Ringtechnik nach $\mathrm{R} u$ t $\mathrm{t}$ e $\mathrm{r}$ (1948), - modifiziert von A. Seifer und O. Oreskes (1953) sowie von M. Wiew iórowski (unveröff.). Am häufigsten wenden wir Rundfilter $(\varnothing 11 \mathrm{~cm})$ an, die eine parallel zur Papierfaserrichtung ausgeschnittene "Zunge“" $(4 \times 40 \mathrm{~mm})$ besitzen. Auf diese Chromatogramme bringen wir eine Lösung auf den Mittelpunkt, oder, wenn nach der Technik von K. V. G i r i und N. A. N. R a o (1952) gearbeitet wird, maximal 7 Lösungen. Weitere Einzelheiten über die angewandte chromatographische Technik beschreiben wir im experimentellen Teil dieser Arbeit. 
B. DIE ZUM ENTWICKELN ANGEWANDTEN LOSUNGSMITTELGEMISCHE

Verhältnismässig leicht konnten wir die Zusammensetzung der Lösungsmittelgemische feststellen, die eine deutliche Trennung des Lupanins, Sparteins und Hydroxylupanins erlaubten. Das erste von uns angewandte Lösungsmittelgemisch war die von R. Munier und Mitarbeitern (1949-52) angegebene Mischung: n-Butanol — Essigsäure mit Wasser gesättigt. Diese Mischung war jedoch ungeignet weil ihre Zusammensetzung wegen Esterbildung dauernd Aenderungen unterlag, die auf die Trennwirkung grundsätzlichen Einfluss hatten. Unter Berücksichtigung dieser Tatsache, als viel brauchbarer zeigte sich die ebenfalls von R. Munier und Mitarbeitern (1949-1952) vorgeschlagene Mischung in der statt Essigsäure Salzsäure zur Anwendung kam. Diese Mischung, obwohl viel haltbarer als die vorher erwåhnte, besass jedoch ebenfalls Unzulänglichkeiten - wobei die Entmischung (phénomène de démixion) während des Entwicklungsprozesses als besonders störend bemerkt wurde. Eine mit 0,5 m-Kaliumchlorid ausgeführte Impregnation des Filtrierpapiers bewirkte zwar eine bedeutende Einschränkung der Entmischung - konnte jedoch diese nicht völlig beseitigen. Als besonders geeignetes Lösungsmittel fanden wir n-Butanol mit Wasser gesättigt, dessen Vorteile man in folgenden Punkten zusammenfassen kann:

1. Der Verbrauch an Lösungsmittel ist minimal - er beträgt nur soviel, wie für das Entwickeln eines Chromatogramms benötigt wird. Der Ueberschuss an Lösungsmittel, der nach dem Entwickeln eines Chromatogramms zurückbleibt, kann nach wiederholtem Ausschütteln mit Wasser unmittelbar weiter angewendet werden.

2. Die Zusammensetzung des Lösungsmittels ist relativ konstant (sie hängt lediglich von der Temperatur und nicht von einer fortschreitenden Esterbildung ab).

3. Das Filtrierpapier muss nicht mit Kaliumchlorid impregniert werden, da eine Aufteilung des Lösungsmittels nicht stattfindet.

4. Die Trennung der Alkaloide ist sehr deutlich - es muss jedoch zwei oder dreimal entwickelt werden.

Auf Grund dieser Vorteile wandten wir in unserer Arbeit hauptsächlich wassergesättigten n-Butanol an.

Das in letzter Zeit von H. Thies und F. W. Reuther (1954) beschriebene Lösungsmittelgemisch, welches zur Trennung des Papaverins und Narkotins dient, besitzt für unsere Zwecke keine besonderen Eigenschaften. Riesige Schwierigkeiten hatten wir bei der Auswahl eines geeignetien Lösünsmittelgemisches zur Trennung des Lupanins vom Lupinin zu überwinden. Es ist eine seltsame und besonderer Berücksichtigung werte Erscheinung, dass die vierringigen Lupinenalkaloide von sehr 
ähnlicher Konstitution: „Lupanin, Spartein und Hydroxylupanin“ (siehe Abb. 1) - ohne weiteres chromatographisch getrennt werden können während sich das zweiringige Lupinin nur unter Anwendung besonderer Lösungsmittelgemische (uns sind nur 2 bekannt) vom vierringigen Lupanin chromatographisch trennen lässt.

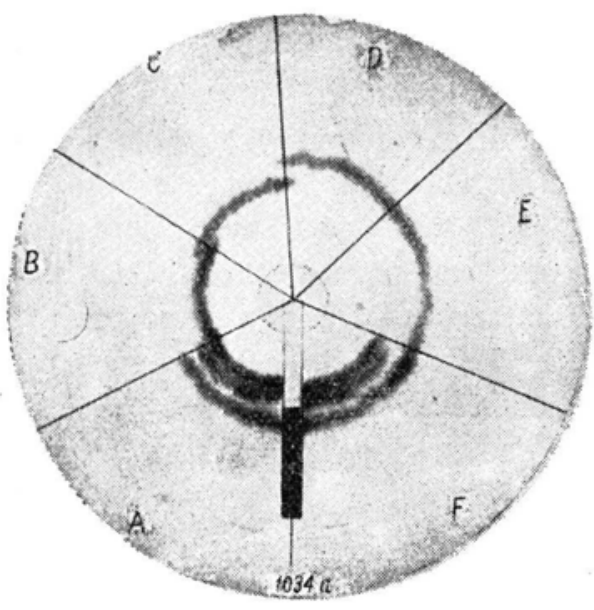

Abb. 2. Lösungsmittelsystem: wassergesättigter n-Butanol (zweimal entwickelt); Entwicklungsreagens. Reagens mit Phosphormolybdänsäure nach P. H. L is t B. Spartein-hydrochlorid, C. Hydroxylupanin-hydrochlorid, D. Lupanin-hydrochlorid, E. Lupinin-hydrochlorid, A und F. Mischung von $\mathrm{Hy}-$ drochloriden aller oben genannten vier Basen.

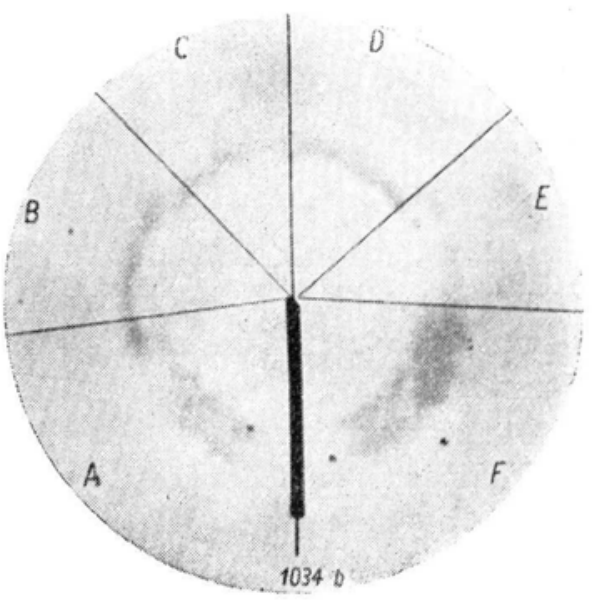

Abb. 3. Lösungsmittelsystem: 4-m-Ammoniumsulfatlösung mit Aethanol (15:1 $\mathrm{v}: \mathrm{v})$ (einmal entwickelt), Entwicklungsreagens: Modifiziertes $\mathrm{D} r$ a $\mathrm{g}$ e $\mathrm{n}$ d o r f f - Reagens, B. Spartein-hydrochlorid, C. Hydroxylupanin-hydrochlorid, B. Lupanin-hydrochlorid, E. Lupinin-hydrochlorid, A und F. Mischung aller vier oben genannten $\mathrm{Ba}-$ sen.

Eines dieser Lösungsmittelgemische besteht aus: Essigsäureäthylester, tert.-Butanol und Wasser. Die Mischung erlaubt eine Trennung aller vier genannten Alkaloide - sie ist jedoch schwer regenerierbar und weist nur kleine Unterschiede in den erhaltenen Rf-Werten auf. Die letztere Erscheinung war der Grund dafür, dass die Mischung nur beschränkt Anwendung fand. Lediglich in Lösungen von ausgeglichener Alkaloidzusammensetzung konnten befriedigende Ergebnisse gefunden werden. Pflanzenextrakte weisen jedoch fast nie eine solche Alkaloidzusammensetzung auf.

Das zweite uns bekannte Lösungsmittel, das die Trennung des Lupinins vom Lupanin ermöglicht, ist eine $4 \mathrm{~m}$-Ammoniumsulfatlösung. Dieses Lösungsmittel trennt deutlich Lupinin vom Lupanin - versagt jedoch bei der Trennung des Lupinins vom Spartein und ebenfalls bei der Trennung des Lupanins vom Hydroxylupanin. 
A. R e s p l o n d y (1955), der dieses Lösungsmittel zur Trennung bestimmter Alkaloide und Aminosäuren benutzte, stellte fest, dass konstitutionsähnliche Substanzen auf Chromatogrammen ähnliche — oder gar gleiche Plätze einnehmen, und zog daraus den Schluss, dass Ammoniumsulfatlösung eine grosse Bedeutung bei der Ermittlung der Konstitution unbekannter Verbindungen haben könnte. Unsere Feststellung, dass Lupinin und Spartein - also Verbindungen verschiedener Konstitution auf Chromatogrammen die gleiche Stelle einnehmen, widerspricht der empirischen Hypothese von R. R e s plond y (1955). Es sei denn, dass das Vorkommen des Lupinins in zweimolekularer Verbindung annehmbar wäre, was schon O. A chmatowicz und W. Rodewald (1955) im Falle von Annotoxin bemerkten. Das merkwürdige Verhalten des Lupinins auf Chromatogrammen ist Gegenstand besonderer Untersuchungen, über die wir an anderer Stelle berichten wollen. Das zuletzt erwähnte Lösungsmittel ist - obwohl es keine deutliche Trennung gibt für unseren Zweck sehr wertvoll (Abb. 3). Nach seiner Anwendung und zusätzlicher Anwendung von wassergesättigtem n-Butanol hatten wir nämlich die Möglichkeit, mit Hilfe zweier verschiedener Chromatogramme, alle vier uns interessierenden Alkaloide zu trennen (Abb. 2 u. 3).

\section{TRENNUNG DER FREIEN ALKALOID-BASEN UND IHRER SALZE}

Eine Reihe von Einführungsversuchen waren, unter anderem, der Form, in welcher die Alkaloide auf das Filtrierpapier zu bringen wären, gewidmet. Es handelte sich hier hauptsächlich darum, ob freie Alkaloidbasen oder ihre Salze zur Anwendung kommen sollten. Es konnte festgestellt werden, dass man bei Anwendung der Hydrochloride des Lupanins, Hydroxylupanins und Lupinins etwas markantere Ringe erhält im Grunde genommen war es jedoch gleich, ob freie Alkaloidbasen oder ihre Salze aufgetragen wurden. Anders verhält sich jedoch das Spartein, welches, in Abhängigkeit von der Form (freie Base - Salz) und dem pH-Wert der Entwicklungsmischung, verschiedene Rf-Werte aufweist und sich unter Umständen sogar in zwei Ringe aufteilen kann (Abb. 4).

Ein solches Verhalten des Sparteins stimmt mit den Ansichten von R. M un i e r und Mitarb. (1940-1952) sowie A. P. B u r m a (1954) überein, die ein solches Phänomen bei einigen Alkaloiden feststellen konnten. Dieses Verhalten ist wahrscheinlich dadurch bedingt, dass die gleiche Substanz drei verschiedene Stellen des Chromatogramms einnehmen kann - als freie Base, als dissoziiertes Salz und als undissoziiertes Salz. Die erwähnte Anormalität findet nicht statt, wenn wir das Spartein in Form ihres Hydrochlorids auf das Filterpapier bringen und neutrale oder saure Lösungsmittel zum Entwickeln anwenden. Zufällig stellten wir je- 
doch fest, dass sogar bei der zuletzt erwähnten Arbeitsweise einige Störungen auftreten können, die in Form zwei verschiedener Ringe in Erscheinung treten. Dieses Verhalten wurde von uns immer dann bemerkt, wenn die Luft des Arbeitsraumes zufällig Dämpfe einer flüchtigen Base wie Ammoniak oder sogar der sehr schwachen Base, Pyridin - enthielt.
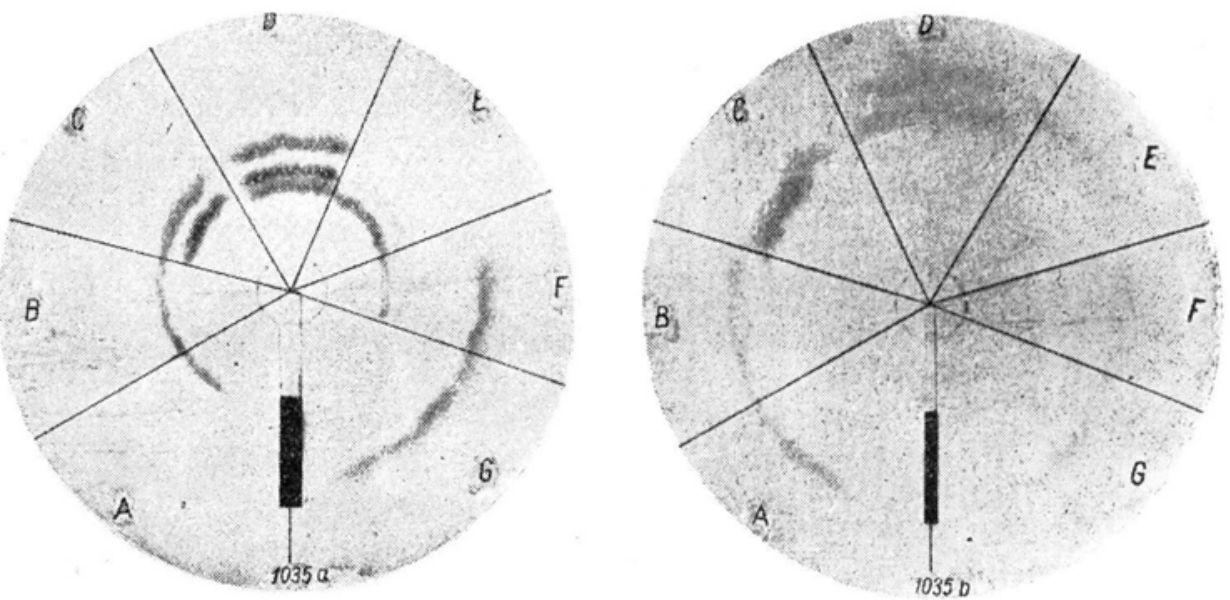

Abb. 4. Auf beide ${ }^{\circ}$ Rundfilter wurden die gleichen Lösungen gebracht, nämlich: A. Lupanin-(Base); B. Lupanin-hydrochlorid; C. Hydroxylupanin- und Lupanin-Base; E. Spartein-hydrochlorid; F. Spartein-acetat; G. Spartein-(Base); D. Mischung von Hydrochloriden aller oben genannten Basen.

Das Chromatogramm 1035a wurden zweimal mit wassergesättigtem n-Butanol entwickelt und die Alkaloide mit dem Phosphormolybdänsäure - Reagens nach P. H. List sichtbar gemacht. Das Chromatogramm $1035 \mathrm{~b}$ wurde einmal mit 4-m-Ammoniumsulfatlösung - Aethanol (15:1 v:v) entwickelt und die Alkaloide mit Dragendorff-Reagens sichtbar gemacht

Je nach der Konzentration der alkalischen Dämpfe der Luft, erfährt ein grösserer oder kleinerer Teil des Sparteinhydrochlorids eine Umsetzung in freie Base, welche nach Entwickeln des Chromatogramms an einer anderen Stelle als das ursprüngliche Hydrochlorid erscheint. Um derartige Störungen zu beseitigen, tragen wir auf den Punkt auf den wir die Lösung des Alkaloidhydrochlorids brachten noch zusätzlich eine „Oese“ 0,5 n-Salzsäure auf.

\section{DIE ENTWICKLUNGSREAGENTIEN}

Für qualitative Zwecke eignet sich am besten das von R. M u n i e r und Mitarb. (1949, 1951, 1952) modifizierte Drag en d orff-Reagens. Von ungefär gleicher Empfindlichkeit sind das Reagens mit Thaliumacetat (R. M unier u. Mitarb. 1949, 1951, 1952) und das Reagens mit Phosphor-Molybdänsäure (P. H. L is t 1954). Die erwähnten Reagentien weisen grosse Empfindlichkeit gegenüber Lupinenalkaloiden auf, so konn- 
te z. B. ein Fleck $\varnothing 2 \mathrm{~mm}$, der 0,001 $\mu \mathrm{g}$. Spartein enthielt, gefärbt werden. Eine grosse Unzulänglichkeit aller genannten Reagentien ist jedoch der Empfindlichkeitsunterschied in Bezug auf verschiedene Alkaloide.

Zusätzlich stellten wir fest, dass mit Dragendorff-Reagens getränktes Filtrierpapier als gut haltbares Indicatorpapier für wässrige Alkaloidlösungen Anwendung finden kann. Dieses Indicatorpapier nannten wir Dragendorff-Papier. Ein Tropfen wässriger Alkaloidlösung, auf das Dragendorff-Papier gebracht, bildet einen runden Fleck oder Ring, der rosa bis rot gefärbt ist.

DIE AUSWAHL DES VERFAHRENS ZUR EXTRAKTION DER ALKALOIDE AUS PFLANZENMATERIAL

Nach Bearbeitung eines entsprechenden chromatographischen Verfahrens begannen wir mit der Analyse der Lupinensamen. In unserer Arbeit beschäftigen wir uns ausschliesslich mit L u p in en s a m e n und besonders mit Samen der schmalblättrigen und weissen Lupine. Vor Beginn der eigentlichen Analyse der Lupinenalkaloide musste ein Extraktionsverfahren ausgewählt werden.

Die Literatur beschreibt zwei Typen von Extraktionsverfahren der Lupinenalkaloide, die Alkoholextraktion (neutrale oder saure) und die alkalische Chloroformextraktion (oder Chloroform-Aetherextraktion). Anfangs arbeiteten wir nach der Alkoholextraktion von P. Bellet (1950) und F. G a l in ovsk y (1951). Die aus gleichem Pflanzenmaterial erhaltenen Rohextrakte wiesen auf Chromatogrammen ausser Lupanin und Hydroxylupanin noch 3 bis 4 Substanzen auf, die ebenfalls mit dem Dragendorff-Reagens reagierten. Die Chromatogramme mit der von F. Galinovsky beschriebenen Methode erhaltenen Rohextrakte unterschieden sich jedoch deutlich von denen mit Extrakten nach P. B e 11 e t. Eine genaue Untersuchung der beiden Extraktionsverfahren zeigte, dass in den einzelnen Arbeitsabschnitten gewisse Verluste von verschiedener Quantität und Qualität an Alkaloidsubstanz vorkommen. Daraufhin beschlossen wir, die von $\mathrm{Z}$. W i e r z c how s ki und L. O b uchow sk a (1952) zur quantitativen Analyse der Lupinenalkaloide vorgeschlagene alkalische Extraktion zu versuchen. Einführende Untersuchungen der Methode bewiesen, dass das Verfahren der Autoren, nämlich die gemahlenen Lupinensamen nach Alkalisierung mit einem wasserbindenden Mittel zu trocknen (Alabastergips), richtig sind.

Genauere Untersuchungen zeigten jedoch, dass ein einmaliges Ausschütteln des vorbereiteten Materials mit Aether-Chloroformmischung so, wie es die Autoren vorschlagen - nicht zu einer quantitativen Alkaloidextraktion führt. Das Verfahren von Z. W i e r z c h o w s ki u. Mitarb. 
(1952) wurde von uns mit der Abänderung nach E. Graf (1952) ergänzt - das alkalisierte, und mit Gips getrocknete Lupinenmehl wurde in Chromatographieröhrchen gebracht (Ø 1,5; h $25 \mathrm{~cm}$ ) und die Alkaloide mit Chloroform ausgewaschen. Im Laufe weiterer Arbeiten ersetzten wir den von $\mathrm{Z}$. W i e r z chowski vorgeschlagenen Alaba-
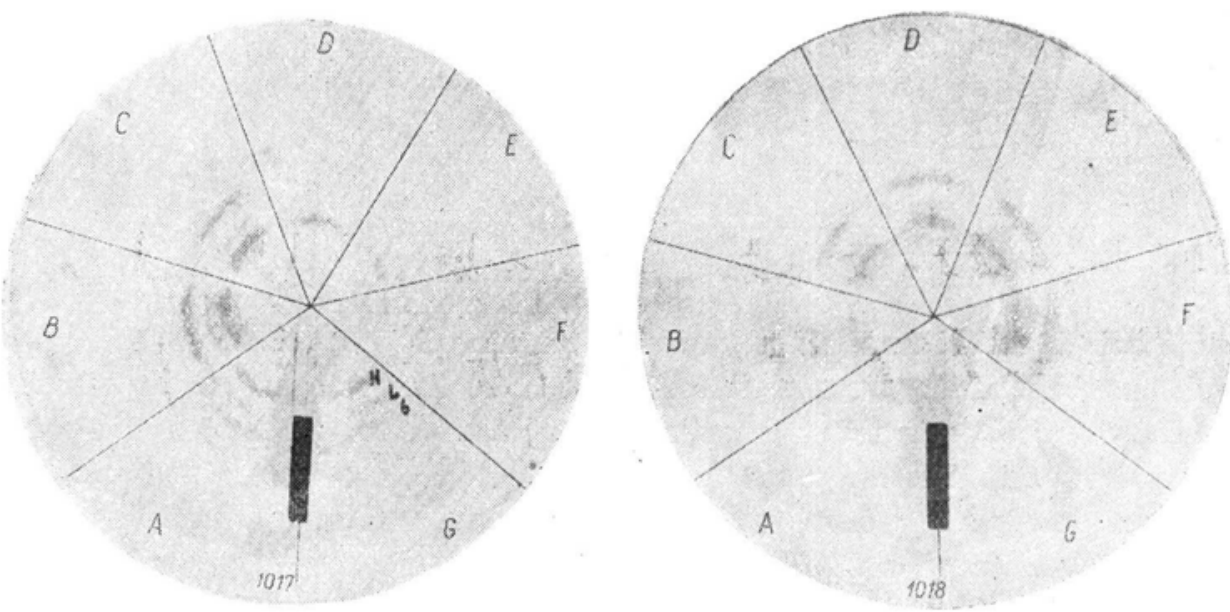

Abb. 5. Beide Chromatogramme wurden zweimal mit wassergesättigtem n-Butanol entwickelt und die Substanzen mit Dragendorff-Reagens gefärbt. Auf beide Chromatogramme wurde in Position A und G eine Vergleichslösung aufgetragen, die aus Hydrochloriden des Hydroxylupanins (H), Lupanins (L), und des ,Alkaloids" $\mathrm{n}_{6}$ (6) bestand. Auf die übrigbleibenden Plätze wurden Alkaloidextrakte aus schmalblättriger (blau,-bitter) Lupine aufgetragen. Proben von 1 g gemahlener Lupinensamen wurden mit gesättigter Natriumcarbonatlösung alkalisiert und mit verschiedenen Mengen trockenem Natriumcarbonat getrocknet. Für Probe B wurde ein $g$ Natriumcarbonat zum Trocknen angewendet, für Probe $\mathrm{C}-1,5 \mathrm{~g}$, für Probe D $2 \mathrm{~g}$, für Probe $\mathrm{E}-2,5 \mathrm{~g}$ und für Probe F $3 \mathrm{~g}$. Die so vorbereiteten Proben wurden erst gründlich mit Chloroform (- Chromatogramm 1017), und dann mit $10 \%$ Methanol in Chloroform (Chromatogramm 1018) extrahiert

ster-Gips durch wasserfreies Natriumsulfat. Wir nahmen an, dass unsere Arbeitsweise fehlerfrei ist, und zu einer fast restlosen Extraktion der Alkaloide aus den Lupinensamen führt.

Die in der Zwischenzeit veröffentlichte Arbeit von J. Reiffer und M. Moż ejko (1954) veranlasste uns, sich nochmals mit dem Extraktionsverfahren zu befassen. Die genannten Autoren widmen in ihrer Arbeit über die qualitative Alkaloidbestimmung in der Lupine der Alkaloidextraktion sehr viel Aufmerksamkeit. Nach unseren Untersuchungen führt das von I. R e if e r und M. M o ż e jko angegebene Extraktionsverfahren nicht zu einer quantitativen Alkaloidisolierung. Nähere diesbezügliche Bemerkungen bringen wir in einer Arbeit, die der quantitativen Analyse der Lupinenalkaloide gewidmet sein soll. Die Autoren unterstreichen jedoch mit Recht die Schwierigkeiten, die bei der Extrak- 
tion der Lupinenalkaloide auftreten und durch vortreffliche Lössbarkeit der Alkaloide in Wasser (ausser Spartein) sowie in organischen Lösungsmitteln bedingt sind. Während weiterer Extraktion und Reinigung der Lupinenalkaloide bemerkten wir, dass die für die Trocknung des Lupinenmehlmacerats angewandten Trockenmittel einen grossen Teil der Alkaloide festhalten und somit ihre völlige Extraktion mit Chloroform oder Aether-Chloroformmischung unmöglich machten. Dieses betrifft sowohl den Gips, wie das wasserfreie Natriumcarbonat und ebenfalls das von uns (1956) angewandte Natriumsulfat.

Das mit Natriumcarbonatlösung (oder Natriumlauge) alkalisierte Lupinenmehl enthält nach Trocknung mit einem der 3 oben genannten Trockenmittel und Extraktion mit Chloroform weiterhin noch grosse Mengen Alkaloide - ja sogar noch dann, wenn wir eine zehnfache Menge des vorgeschriebenen Chloroforms anwandten. Dieses bezieht sich ebenso auf Extraktion durch Ausschütteln, sowie auf Auswaschen der Alkaloide aus der Chromatographiesäule. Eine nach ausführlicher Chloroformextraktion folgende Extraktion mit Methanol oder mit Methanol-Chloroformmischung ergibt weitere Alkaloidfraktionen, die eine ähnliche qualitative Alkaloidzusammensetzung wie die Chloroformfraktionen aufweisen. Eine schätzungsweise Bewertung der Chromatogramme berechtigt uns zu der Annahme, dass je mehr Natriumcarbonat zum Trocknen des Lupinenmehls angewendet wird, um so weniger Alkaloide eluiert werden. Das Chromatogramm auf Abb. 5 zeigt uns diesen Zusammenhang.

Da der Methanol-Chloroformextrakt (erhalten nach ausführlicher Extraktion mit reinem Chloroform) ausser Alkaloiden auch eine Menge Lipide enthält, die in Methanol löslich sind, nahmen wir anfangs an, dass diese eine völlige Extraktion der Alkaloide unmöglich machen. indem sie die Alkaloide okkludieren und somit das Chloroform nicht hinzulassen. Um diese Hypothese zu überprüfen führten wir mit der bitteren, weissen, schmalblättrigen und mit der gelben Lupine folgende Versuche aus:

Die gemahlenen Lupinensamen wurden teilweise mit wasserfreiem Aceton durch mehrmaliges Ausschütteln entfettet, mit Natriumcarbonatlösung alkalisiert und mit wasserfreiem Natriumcarbonat getrocknet. Nachher wurden sie in Erlenmeyer-Kolben gebracht und nacheinander mit folgenden Lösungsmitteln ausführlich extrahiert: 1) Petroläther, 2) Cyclohexan, 3) Chloroform, 4) 10\% Methanollösung in Chloroform. Die Chromatogramme auf Abb. 6 stellen die Alkaloidzusammensetzung der einzelnen Fraktionen dar, wobei die Alkaloide, die bei der Entfettung in Lösung gegangen sind, ebenfalls angeführt wurden.

Aus diesen Chromatogrammen ist ersichtlich, dass die Hypothese, die ein Zurückhalten der Alkaloide durch anwesende Lipide annimmt, falsch 
ist. Unter vielen, von uns untersuchten Trockenmitteln, besass Kieselgur die besten Eigenschaften. Nach Trocknen mit Kieselgur konnten wir nämlich die Gesamtmenge an Alkaloiden mit reinem Chloroform extrahieren. Bei Anwendung von Kieselgur zur Extraktion der schmalblättrigen Lupine stellten wir fest, dass auch eine fast quantitative Trennung des Lupanins und des ,Alkaloids“ $n_{3}$ vom Hydroxylupanin und dem „Alkaloid“ $\mathrm{n}_{6}$ möglich ist. Dieses Verhalten beobachteten wir, wenn wir
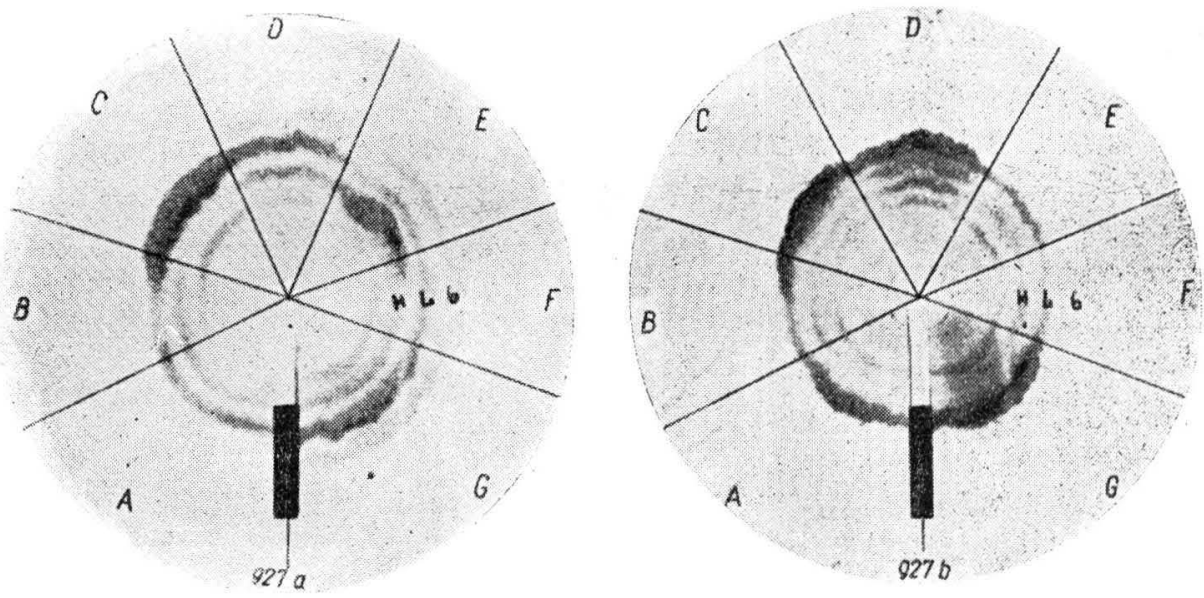

Abb. 6. Die Chromatogramme wurden zweimal mit wassergesättigtem n-Butanol entwickelt und die Substanzen mit Dragendorff-Reagens gefärbt. Das Chromatogramm $\mathrm{Nr}$ 927-a betrifft die schmalblättrige, das Chromatogramm Nr. 927-b betrifft die weisse und das Chromatogramm Nr. 927-c betrifft die gelbe Lupine.

In Position $\mathrm{B}$ und $\mathrm{F}$ wurden Vergleichslösungen aufgetragen, die aus den $\mathrm{Hy}$ drochloriden des Hydroxylupanins $(\mathrm{H})$. des Lupanins (L) und des ,Alkaloids $n_{6}$ (6) bestehen.

In Position A wurde angesäuerte, aus dem Acetonextrakt erhaltene Lösung, aufgetragen - in Position C - Petrolätherextrakt, in Position D - Cyclohexanextrakt, in Position $\mathrm{E}$ - Chloroformextrakt und in Position G - Extrakt mit $10 \%$ Methanol in Chloroform.

das alkalisierte und mit Kieselgur getrocknete Lupinenmehl in Chromatographieröhrchen brachten und die Alkaloide mit Petroläther (Siedep. $35^{\circ}$ bis $60^{\circ} \mathrm{C}$ ) und nachher mit Chloroform ausführlich extrahierten. Der Petrolätherextrakt enthielt Lupanin und das „Alkaloid“ $\mathrm{n}_{6}$, während der Chloroformextrakt Hydroxylupanin und das „Alkaloid“ $\mathrm{n}_{6}$ aufwies. 
Die Chromatogramme auf Abb. 7 stellen die fraktionierte Auswaschung der Alkaloide aus Lupinenmehl dar.

Aehnliche Eigenschaften wie Kieselgur besitzt auch Calciumsilicat. Während dem Suchen nach einem geeigneten Trockenmittel bemerkten wir, dass die Fuller-Erde stark adsorbierende Eigenschaften in Bezug auf die Lupinenalkaloide besitzt. Eine langdauernde Extraktion des alkalisierten und mit Fuller-Erde getrockneten Lupinenmehls führte zu keiner bemerkenswerteren Isolierung der Alkaloide. Im Laufe der Untersu-
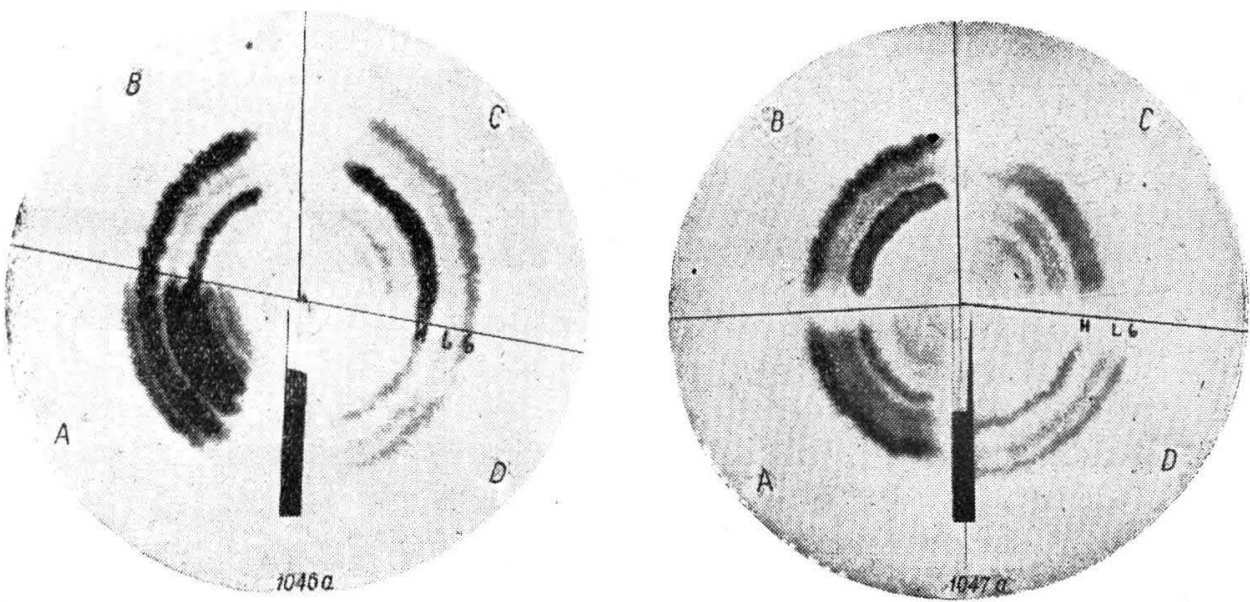

Abb. 7. Die Chromatogramme wurden dreimal mit wassergesättigtem n-Butanol entwickelt und mit dem Phosphormosäure- Reagens nach P. H. List entwickelt. Chromatogramm Nr. 1046 betrifft die schmalblättrige, und das Chromatogramm Nr. 1047 die weisse Lupine.

In Position D wurde die Vergleichslösung, die aus Hydrochloriden des Hydroxylupanins $(\mathrm{H})$, Lupanins (L) und des „Alkaloids“ $\mathrm{n}_{6}(6)$ besteht, aufgetragen; in Position A unverarbeiteter Chloroformextrakt (Gesamtalkaloide), in Position B - Petrolätherextrakt (Fraktion I), in Position $\mathbf{C}$ der nach dem Petroläther folgende Chloroformextrakt (Fraktion II)

chungen über die Extraktionsverfahren der Lupinenalkaloide beschäftigten wir uns ebenfalls mit dem Vergleich verschiedener zur Alkalisierung der Lupinenmehle dienenden Reagentien. Wir untersuchten Natriumlauge verschiedener Konzentration, gesättigte Natriumcarbonatlösung und Ammoniaklösung. Das beste Alkalisierungsmittel ist Ammoniaklösung - bei ihrer Anwendung erhielten wir Chromatogramme, die schätzungsweise die grösste Alkaloidmenge aufwiesen. Das Aufnehmen, der nach Abdestillieren des Chloroforms zurückbleibenden Alkaloide in angesäuertes Wasser, ist ebenfalls am einfachsten und die auf diese Weise erhaltenen Extrakte sind von grosser Reinheit.

Das schlechteste Alkalisierungsmittel war Natriumlauge und eine Mittelstellung nahm Natriumcarbonatlösung ein. Die genannten Beobach- 
tungen sind unserer Ansicht nach folgendermassen zu erklären: Natriumlauge und Natriumcarbonatlösung bewirken nicht nur eine Befreiung der Alkaloidbasen, sondern hydrolisieren auch teilweise die anwesenden Lipide und Eiweissstoffe. Deshalb ist auch der nach dem Abdestillieren des Chloroforms und Ansäuern zurückbleibende Extrakt meistens getrübt, schwerfiltrierbar und nach weiterem Einengen bräunlich gefärbt, wobei man noch gelegentlich eine Niederschlagsbildung beobachten kann. Eine solche Lösung bildet auf dem Startpunkt des Chromatogrammes einen dunklen Fleck, und die Trennung der Alkaloide ist nicht scharf. Das Alkalisieren des Lupinenmehles mit Ammoniaklösung beseitigt grösstenteils die genannten Störungen.

DIE QUALITATIVE ALKALOIDZUSAMMENSETZUNG DER WEISSEN, SCHMALBLÄTTRIGEN UND GELBEN LUPINE - FESTGESTELLT MIT HILFE. DER PAPIERCHROMATOGRAPHIE

Die qualitative Analyse zeigte, dass in der Lupine ausser Lupanin und Hydroxylupanin noch andere alkalisch reagierende Substanzen anwesend sind, die mit den Alkaloidreagentien positiv reagieren. Das auf Abb. 8 dargestellte Chromatogramm zeigt die Alkaloidzusammensetzung von bitteren Biotypen der drei angeführten Lupinenarten.

Die bisher unbekannten „Alkaloide“ nannten wir, nach Berücksichtigung ihrer Verteilung auf dem Chromatogramm, nach steigernden $R_{f}$ Werten: $\mathrm{n}_{1}, \mathrm{n}_{2}, \mathrm{n}_{3}, \mathrm{n}_{4 / 5}$ und $\mathrm{n}_{6}$ (bei Anwendung von wassergesättigtem n-Butanol). Hydroxylupanin liegt auf dem Chromatogramm zwischen $\mathrm{n}_{3}$ und $n_{4 / 5}$ während Lupanin den Platz zwischen $n_{4 / 5}$ und $n_{6}$ einnimmt. An Hand des abgebildeten Chromatogramms ist ersichtlich, dass jede der drei angeführten Lupinénarten eine bestimmte Alkaloidzusammensetzung besitzt.

Die weisse Lupine enthält hauptsächlich Lupanin, neben dem noch das ,Alkaloid“ $\mathrm{n}_{3}$, Hydroxylupanin und das „Alkaloid“ $\mathrm{n}_{1 / \mathrm{s}}$ anzutreffen ist. Die schmalblättrige Lupine weist hauptsächlich Lupanin und Hydroxylupanin auf. In verhältnismässig grossen Mengen sind auch noch die „Alkaloide“ $\mathrm{n}_{6}$ und $\mathrm{n}_{3}$ anwesend, während die ,Alkaloide“ $\mathrm{n}_{1}$ und $\mathrm{n}_{2}$ nur in sehr geringen Mengen auftreten. Die mehrjährige Lupine weist eine änliche Alkaloidzusammensetzung auf wie die schmalblättrige — enthält jedoch noch zusätzlich ein Alkaloid, das chromatographisch mit dem Spartein übereinstimmt.

Das beschriebene Chromatogramm (Abb. 8) wurde mit Hilfe nicht verarbeiteter Alkaloidextrakte der drei angeführten Lupinenarten hergestellt. Wenn die Alkaloide aus der weissen Lupine zuerst mit Petroläther und nachher mit Chloroform extrahiert werden, kann man in dem Chloroformextrakt zusätzlich noch das ,Alkaloid“ $\mathrm{n}_{6}$ und Spuren der „Alka- 
loide" $n_{1}$ und $n_{2}$ finden. Die qualitative Alkaloidzusammensetzung ist also für alle drei Lupinenarten sehr ähnlich. Es bestehen jedoch Unterschiede im mengenmässigen Verhältnis der einzelnen Alkaloide, so dass man ohne weiteres an Hand des Chromatogrammes feststellen kann, welche Lupinenart vorliegt. Abb. 9 zeigt ein Chromatogramm, das aus Samen der bitteren, gelben Lupine hergestellt wurde.

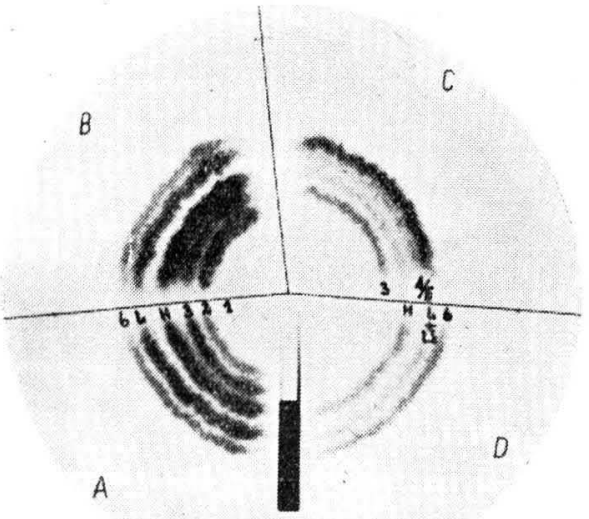

$1044 a$

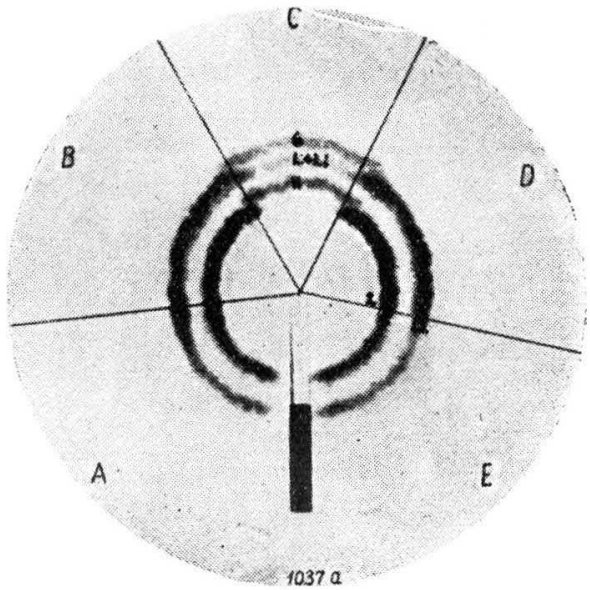

Abb. 9. Das Chromatogramm wurde zweimal mit wassergesättigtem n-Butanol entwickelt und mit Phosphormolybdänsäure-Reagens nach $\mathrm{P}$. $\mathrm{H}$. Lis t gefärbt. A und $\mathrm{E}-$ aus Hydrochloriden des Sparteins (S) und Lupinins (Li) bestehende Vergleichslösung; $B$ und $D$ - unverarbeiteter Alkaloidextrakt aus der gelben Lupine; C aus Hydrochloriden des Hydroxylupanins $(\mathrm{H})$, Lupanins (L), Lupinins (Li) und des "Alkaloids" $\mathrm{n}_{6}$ (6) bestehende Vergleichslösung

Wir sehen daraus, dass die Alkaloidzusammensetzung der gelben Lupine viel weniger kompliziert ist als die der bisher besprochenen Lupinenarten. Es liegt hier hauptsächlich Lupinin und Spartein vor, während die Ringe der „Alkaloide“ $\mathrm{n}_{2}$ und $\mathrm{n}_{3}$ nur schwach gefärbt sind.

Die auf Abb. 8 und 9 dargestellten Chromatogramme wurden mit wassergesättigtem n-Butanol entwickelt - sie konnten also die Anwesenheit des Lupinins neben Lupanin nicht aufweisen. Aus diesem Grunde stellten wir zusätzlich noch zwei Chromatogramme her und entwickelten sie mit 4-m-Ammoniumsulfatlösung (sie trennt Lupinin von Lupanin).

Nach Betrachtung des auf Abb. 10 dargestellten Chromatogramms konnten wir feststellen, dass in der schmalblättrigen und mehrjährigen 
Lupine in kleinen Mengen auch Lupinin vorkommt, und in der gelben Lupine Spuren von Lupanin beziehungsweise Hydroxylupanin anzutreffen sind. Unsere bisherige Erkenntnis auf dem Gebiete der qualitativen Alkaloidzusammensetzung der genannten Lupinenarten stellten wir in Form einer Tabelle dar, die ebenfalls die $\mathrm{R}_{\mathrm{f}}$-Werte für die einzelnen Alkaloide enthält.

\section{$\mathrm{T}$ a b e $11 \mathrm{e} 1$}

Die qualitative Alkaloidzusammensetzung der weissen, schmalblättrigen, mehrjährigen und gelben Lupine

\begin{tabular}{|c|c|c|c|c|c|c|}
\hline \multirow[b]{2}{*}{$\begin{array}{l}\text { Name des } \\
\text { Alkaloids }\end{array}$} & \multicolumn{2}{|c|}{$\begin{array}{l}\text { Rf }- \text { Werte für das } \\
\text { Lösungsmittelsystem: }\end{array}$} & \multicolumn{4}{|c|}{ Lupinenart $* *$} \\
\hline & $\begin{array}{c}\text { wassergesät- } \\
\text { tigter n-Buta- } \\
\text { nol }\end{array}$ & $\begin{array}{c}4 \mathrm{~m}\left(\mathrm{NH}_{4}\right)_{2} \mathrm{SO}_{4} \\
\text { mit Aethanol } \\
(15: 1 \mathrm{v}: \mathrm{v})\end{array}$ & $\begin{array}{l}\text { schmal- } \\
\text { blättrige }\end{array}$ & $\begin{array}{l}\text { mehr- } \\
\text { jährige }\end{array}$ & weisse & gelbe \\
\hline $\mathrm{n}_{1}$ & ungef. 0.15 & - & (X) & (X) & (X) & \\
\hline $\mathrm{n}_{2}$ & , $\quad 0,25$ & 一 & $\mathrm{XX}$ & $\mathrm{xxx}$ & $(\mathrm{X})$ & $\mathrm{X}$ \\
\hline $\mathrm{n}_{3}$ & .. 0,33 & ungef. 0,62 & $\mathrm{XXX}$ & $\mathrm{xx}$ & $\mathrm{XXX}$ & $(\mathrm{X})$ \\
\hline Spartein & $, \quad 0,36$ & 0,76 & & $\mathrm{xx}$ & $(\mathrm{X})$ & $\mathrm{XXXXX}$ \\
\hline Hydroxylupan. & $, \quad 0,42$ & 0,62 & $\mathrm{XXXX}$ & $\mathrm{xxX}$ & $\mathrm{xx}$ & (X) \\
\hline $\mathrm{n}_{4 / 5}$ & , 0,50 & 0,62 & & & $\mathrm{xx}$ & \\
\hline Lupanin & , $\quad 0,55$ & 0,65 & $\mathrm{x} \times \mathrm{XX}$ & $\mathrm{XXXX}$ & $\mathrm{XXXX}$ & $(\mathrm{X})$ \\
\hline Lupinin & $, \quad 0,55$ & 0,76 & $\mathrm{xx}$ & $(\mathrm{X})$ & & $\mathrm{XXXXX}$ \\
\hline $\mathrm{n}_{6}$ & $, \quad 0,65$ & 0,62 & $\mathrm{xxxx}$ & $\mathrm{XXXX}$ & $\mathrm{x}$ & \\
\hline
\end{tabular}

Unsere Ergebnisse zeigen, dass die Alkaloidzusammensetzung der Lupine weit mehr kompliziert ist, als bisher angenommen wurde. Diese Ansicht wird auch von Seiten anderer Autoren bestätigt, die ähnliche Arbeiten ausführten. Im Jahre 1954 erschien eine, die Alkaloidzusammensetzung von Lupinus termis betreffende Arbeit von E. H. W. J. B u r d e n

* In unserer Arbeit bedienten wir uns nicht der $\mathrm{R}_{\mathrm{f}}$-Werte zur chromatographischen Identifizierung der einzelnen Substanzen. Die von uns angewandte Technik erlaubte ohne Schwierigkeiten eine unmittelbare Identifizierung der Substanzen. Auch entwickelten wir die Chromatogramme in den meisten Fällen mehrmals, wobei es schwer ist die Lösungsmittelfront immer auf die gleiche Entfernung vom Startpunkt einzustellen. Die für das Lösungsmittelsystem: wassergesättigter n-Butanol eingetragenen $R_{f}$-Werte wurden nach dreimaligem Entwickeln festgestellt.

** Die Anzahl der X - Zeichen stellt annähernd den mengenmässigen Anteil der einzelnen Alkaloide dar. Ein X - Zeichen in Klammern bedeutet das Auftreten des Alkaloids in Spurenmengen. Das gleiche Zeichen wurde auch dann gesetzt, wenn das Vorkommen eines Alkaloids zweifelhaft war. 
und Mitarb. (1954). Die genannten Autoren konnten mit Hilfe mehrmaliger, selektiver Extraktion und einer grossen Anzahl von Proben und Alkaloidreagentien feststellen, dass die Alkaloidzusammensetzung von Lupinus termis viel komplizierter ist, als dies von G. R. Clemo und G. C. Leitch (1928) angenommen wurde. Die letzteren nahmen an, dass Lupinus termis nur Lupanin enthält - E. H. W. J. Burden und Mitarb. konnten jedoch sieben verschiedene Alkaloide isolieren. Trotz der Verschiedenheit der Arbeitsmethoden, die E. H. W. J. Burden und Mitarb. einerseits und wir anwandten und trotz der Verschiedenheit des Versuchsmaterials konnten ähnliche Ergebnisse erhalten werden.
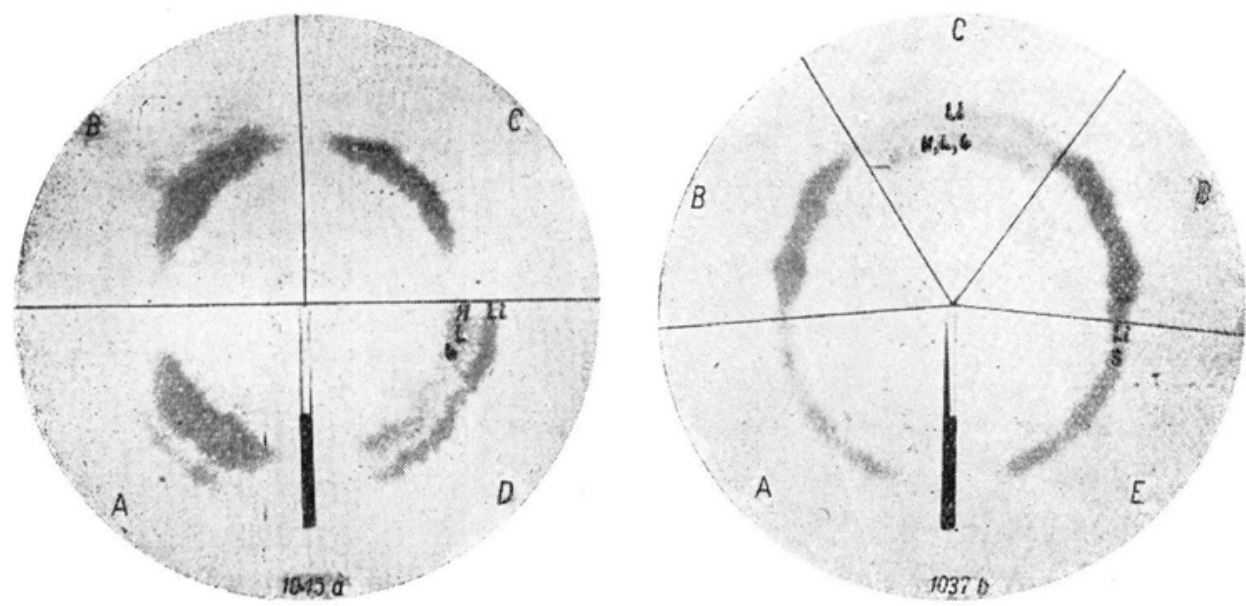

Abb. 10. Das Chromatogramm Nr. 1045-a entspricht genau dem auf Abb. 8 abgebildeten Chromatogramm und das Chromatogramm Nr. 1037-b - dem auf Abb. 9 dargestellten Chromatogramm. Beide Chromatogramme unterscheiden sich von den auf Abb. 8 und 9 dargestellten dadurch, dass sie mit 4-m-Ammoniumsulfatlösung und Aethanol (15:1 v:v) entwickelt wurden und die Alkalo:de mit DragendorffReagens sichtbar gemacht wurden

Die genannten Autoren bemerkten, dass die Alkaloidzusammensetzung komplizierter ist, und dass man bei der Isolierung der einzelnen Alkaloide in Form reiner Kristalle auf grosse Schwierigkeiten stösst - was von uns ebenfalls festgestellt wurde. Es besteht kein Zweifel darüber, dass eine weitere Verwollkommnung der Analyse und Trennung der Lupinenalkaloide zur genaueren und besseren Kenntnis ihrer Zusammensetzung führen wird.

In Anlehnung an ein einheitliches Extraktionsverfahren und eine normalisierte chromatographische Arbeitsmethode begannen wir mit S. B a r b a cki und seinen Mitarbeitern die qualitative Alkaloidzusammensetzung in den Samen vieler Stämme und Biotypen der weissen, schmalblättrigen und gelben Lupine $\mathrm{zu}$ untersuchen. Das Ziel dieser 
Untersuchungen, deren Ergebnisse sukzessiv in den „Roczniki Nauk Rolniczych" (Annalen der Landwirtschaftlichen Wissenschaften) veröffentlicht werden, ist die Klärung der Frage ob die Alkaloidzusammensetzung im Rahmen der einzelnen Biotypen (ew. Stämme) konstant ist und die Feststellung eventueller Zusammenhänge zwischen Alkaloidzusammensetzung und morphologischen Merkmalen der Lupine.

Parallel zu diesen Untersuchungen führten wir eine qualitative chromatographische Analyse der Alkaloide in folgenden Pflanzenteilen aus: in den Wurzeln, Blüten und grünen Teilen der Lupine - in verschiedenen Zeitabschnitten ihrer Entwicklung und unter verschiedenen Anbaubedingungen. Ziel dieser langjährigen Untersuchungen ist der Versuch die Biogenese der Lupinenalkaloide und ihre Funktion zu erfassen. Die ersten Ergebnisse dieser Untersuchungen werden in den „Roczniki Nauk Rolniczych" veröffentlicht werden. An dieser Stelle wollen wir nur mitteilen, dass wir in vielen jungen Pflanzen der weissen Lupine deutliche Mengen an Spartein vorfanden, die im Laufe der Entwicklung abnehmen. Diese Spartein-Abnahme erfolgt, wie es scheint, zu Gunsten des Hydroxylupanins und des „Alkaloids“ $\mathrm{n}_{6}$ deren Menge anstieg.

VERSUCHE ZUR ISOLIERUNG UND IDENTIFIZIERUNG NEUER LUPINENALKALOIDE

Nach uns bekannten Literaturangaben P. B ell e t (1950), T. A. H e nry (1950) und P. H. Lis t (1954) werden die 4 uns interessierenden Alkaloide ausschliesslich durch Destillation bei Unterdruck aus der Mischung der Rohalkaloide isoliert und gereinigt. Nach unserer Meinung ist diese Methode brutal - aus weisser Lupine erhält man auf diese Weise nur Lupanin, aus Lupinus palmeri - nur Lupinin, aus Lupinus polyphyllus erhält man zwar Lupinin und Hydroxylupanin, aber die Alkaloidverluste sind sehr gross, aus Lupinus barbiger erhält man nur Spartein. Aus diesem Grunde beschlossen wir dieses Verfahren zu verwerfen.

Um zu ermitteln ob die ,Alkaloide“ $\mathrm{n}_{1}, \mathrm{n}_{2}, \mathrm{n}_{3}, \mathrm{n}_{4 / 5}$ und $\mathrm{n}_{6}$ nicht vielleicht oft in den Samen der Leguminosen vorkommende Verbindungen wären - führten wir mit Hilfe von Erbsensamen, die nach allgemeiner Meinung keine Alkaloide enthalten, eine gleiche Extraktion und chromatographische Analyse durch, wie im Falle von Lupinensamen. Wenn wir zur Analyse $2 \mathrm{~g}$ gemahlene Erbsensamen (also so viel wie wir gewöhnlich bei der Lupine anwenden) nahmen, so konnten wir auf den Chromatogrammen keinen Alkaloidring feststellen. Erst bei Anwendung von $10 \mathrm{~g}$ - Proben und Aufbringen der Alkaloide in zehnfach grösserer Menge als gewöhnlich - konnten wir auf dem Chromatogramm 2 Ringe feststellen, von denen einer dem Lupanin und einer dem Spartein ent- 
sprach. Aus diesen Untersuchungen geht hervor, dass die von uns neubemerkten „Alkaloide“ $\mathrm{n}_{1} \ldots \mathrm{n}_{6}$ nicht häufig in Leguminosen vorkommende Verbindungen sind und dass die Erbse, die gewöhnlich als alkaloidfrei angesehen wird, wahrscheinlich auch Alkaloide bildet — aber in ca. 50fach kleineren Mengen als süsse Lupinenarten.

Das Hauptobjekt unserer Identifizierungsuntersuchungen war und ist weiterhin die schmalblättrige Lupine, weil sie die am meisten ,,ausgeglichene" Alkaloidzusammensetzung besitzt. Zur präparativen Trennung der Rohalkaloide aus schmalblättriger Lupine wurden von uns 1 . verschiedene papierchromatographische Verfahren, 2. säulenchromatographische Adsorptionsverfahren, 3. säulenchromatographische Verteilungsverfahren, 4. fraktionierte Kristallisation auf Filtrierpapier, sowie, 5. Filtrierpapierelektrophorese angewandt.

Eine ausführliche Beschreibung aller erhaltenen Ergebnisse, sowie der angewandten Trennmethoden veröffentlichen wir an anderer Stelle; hier sollen nur einige von ihnen besprochen werden.

Wie wir schon mitteilten, löst sich das „Alkaloid“ $\mathrm{n}_{6}$ ähnlich wie Lupanin in Petroläther, — das „Alkaloid“ $\mathrm{n}_{6}$ ist in Petroläther, ähnlich wie Hydroxylupanin nicht löslich. Das ,Alkaloid“ $\mathrm{n}_{3}$ "ist mit dem Lupanin, in welches es unter nicht genau zu erfassenden Umständen übergeht, sehr verwandt. Trotz mehrmaligem Wiederholen mit dem präparativen Papierchromatographieverfahren, gelang es uns bisher noch nicht, Lupaninfreies „Alkaloid“ $\mathrm{n}_{3}$ zu erhalten. Ebenfalls versagte die fraktionierte Fällung der Alkaloide aus angesäuerter wässriger Lösung mit Silizium-Wolframsäure oder dem D ragendorff-Reagens. Aus diesen Ergebnissen schlossen wir, dass das sogennante "Alkaloid“ $\mathrm{n}_{3}$ das Di-Hydrochlorid des Lupanins sei, während das Lupanin auf dem Chromatogramm dem Mono-Hydrochlorid entspricht. Diese Annahme mussten wir aber verwerfen, weil wir nach Behandeln reiner Lupaninpräparate mit Aceton, Methanol, Aethanol und konz. Salzsäure immer nur einen Ring auf dem Chromatogramm erhielten, der dem Lupanin entsprach.

Die Hypothese, dass Lupanin bei bestimmter Konzentration mit den zum Entwickeln der Chromatogramme angewandten Lösungsmitteln molekulare Verbindungen bildet, erwies sich ebenfalls als falsch. Diese Hypothese nahmen wir nach Bekanntnahme Arbeit von R. H. H a ckeman und M. La z a r u s (1955) über das Verhalten der Glutaminsäure auf Chromatogrammen, an. Die Vermutung wurde jedoch gegenstandslos weil das in viel grösserer Menge aufgetragene Lupanin auf Chromatogrammen immer nur einen Ring niemals zweie bildete.

Als nächste Hypothese nahmen wir unter Einfluss der Arbeit von M. Rink und K. Grabowski (1955) die folgende an. Die Autoren zeigten unter anderem, dass das 17-Hydroxyspartein in Chloroform gelöst, 
ausserordentlich leicht in 17-Trichlor-Spartein übergehen kann. Nach Berücksichtigung dieser Tatsache nahmen wir an, dass sich das „Alkaloid" $\mathrm{n}_{3}$ während dem Abdestillieren des Chloroforms aus dem Chloroformextrakt bildet. Diese Hypothese musste jedoch verworfen werden, als wir merkten, dass das ,Alkaloid“ $\mathrm{n}_{3}$ auch in Petrolätherextrakten anwesend ist. Gegenwärtig nehmen wir an, dass das „Alkaloid“ $\mathrm{n}_{3}$ eine Verbindung des Lupanins mit einer anderen, bisher noch nicht identifizierten Verbindung ist. Diese Hypothese bedarf gegenwärtig genauerer Utberprüfung. Ebenfalls müssen die Bedingungen, unter welchen das „Alkaloid“ $\mathrm{n}_{3}$ in Lupanin übergeht, noch genau untersucht werden.

Die erbrachten Daten über das Verhalten und die Eigenschaften der „Alkaloide“ $\mathrm{n}_{1}$ und $\mathrm{n}_{2}$ sind noch nicht ausreichend, man darf jedoch wohl annehmen, dass die genanten „Alkaloide“ dem „Alkaloid“ $\mathrm{n}_{2}$ ähnlich sind.

Viel leichter ist die Identifizierung des „Alkaloids“ $\mathrm{n}_{6}$. Dieses „Alkaloid“ besitzt eine grössere Beständigkeit und konnte deshalb mit Hilfe der präparativen Papierchromatographie in Form reiner Kristalle, die auch chromatographisch rein waren, erhalten werden. Die auf diese Weise isolierte Alkaloidmenge (einige $\mathrm{mg}$ ) reichte jedoch nicht aus um eine genaue Identifizierung und Charakterisierung durchzuführen, wies uns jedoch den Weg das Alkaloid, frei von Begleitsubstanzen, zu erhalten.

Einführungsarbeiten über die Isolierung des ,Alkaloids“ $\mathrm{n}_{4 / 5}$ lassen annehmen, dass wir in diesem Falle weniger Schwierigkeiten wie im Falle des ,Alkaloids“ $\mathrm{n}_{3}$ haben werden.

\section{EINFUHRENDE VERSUCHE IN VITRO ZUR BESTIMMUNG DER}

HALTBARKEIT EINIGER LUPINENALKALOIDE SOWIE ZUR BESTIMMUNG DER ZERFALL - UND UMSETZUNGSPRODUKTE

Wir untersuchten: Spartein, Lupanin, Lupinin, Hydroxylupanin und das „Alkaloid“ $\mathrm{n}_{6}$. Wir untersuchten die Umformungs- und Zerfallmöglichkeit der genannten Alkaloide in Form freier Basen sowie in Form ihrer Hydrochloride. Die Arbeitsmethode war einfach. Auf Tüpfelplatten brachten wir je einen Tropfen $2 \%$ Lösung der Basen oder ihrer Hydrochloride, trockneten bei Zimmertemperatur in einem mit Phosphorpentoxyd und Natriumhydroxyd angefüllten Exsikkator und wirkten auf die Proben mit folgenden Reagentien ein: 1. konz. Salzsäure, 2. $3-20 \%$ Wasserstoffperoxyd, 3. konz. Salzsäure und Wasserstoffperoxyd, 4. dest. Wasser, 5. Methanol, 6. Chloroform, 7. konz. Ammoniaklösung. Nach Austrocknen der einzelnen Reagentien (bei Zimmertemperatur) gaben wir zu jedem Fleck je einen Tropfen Wasser und trugen die so bereitete Lösung auf das Chromatogramm auf. Diese Manipulation wiederholten wir eini- 
ge Male. Lupanin und Hydroxylupanin wiesen gegenüber den genannten Reagentien eine völlige Widerstandsfähigkeit auf. Das Spartein unterlag jedoch sehr grossen Veränderungen, die in Form neuer Farbringe auf dem Chromatogramm hervortraten. Besonders das mit Wasserstoffperoxyd behandelte Spartein erfuhr bedeutende Veränderungen. Das Spartein setzte sich unter diesen Bedingungen in eine Verbindung um, die auf dem Chromatogramm an der Stelle erscheint, an welcher gewöhnlich das „Alkaloid“ $n_{1 /,}$ auftritt. Diese Verbindung ist ein Hauptabbauprodukt des Sparteins - ebenfalls in Chloroform- und Aetherlösung. Ausser dieser
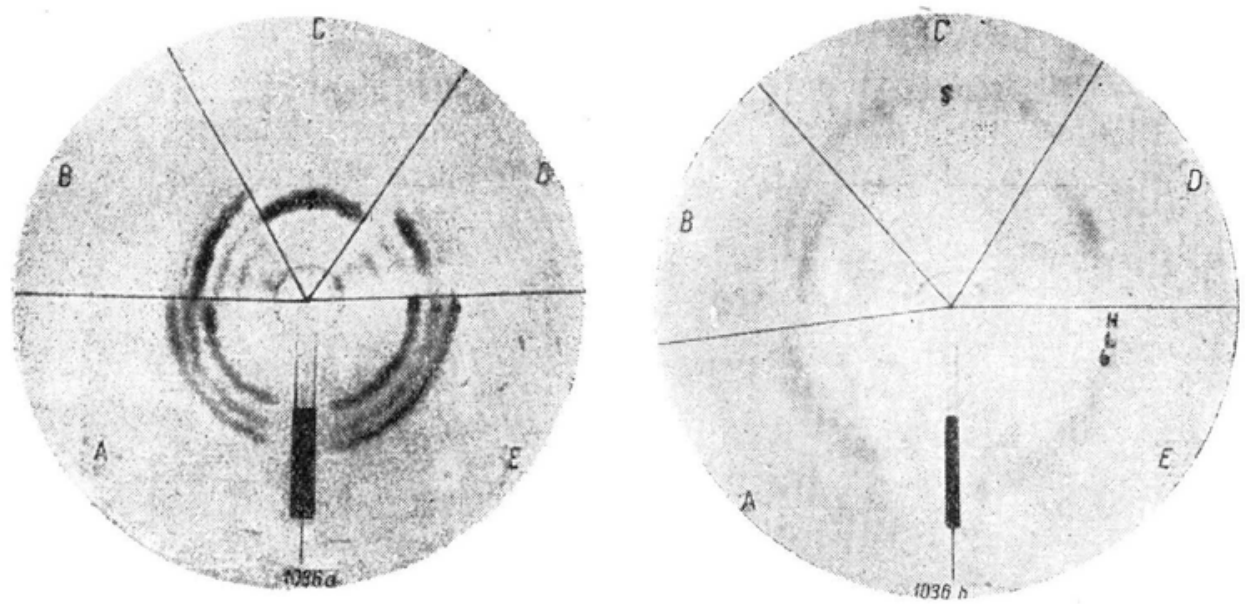

Abb. 11. Auf beide Rundfilter wurde die gleiche Substanz aufgetragen. A und E Vergleichslösung, bestehend aus: Hydrochloriden des Hydroxylupanins $(\mathrm{H})$, Lupanins (L), und des "Alkaloids" $\mathrm{n}_{6}(6)$; C - Spartein-hydrochlorid; B und D - Zersetzungsprodukte der Spartein-(Base). Das Chromatogramm Nr. 1036-a wurde zweimal mit wassergesättigtem $\mathrm{n}-\mathrm{Butanol}$ und das Chromatogramm Nr. 1036-b mit 4-m-Ammoniumsulfatlösung und Aethanol $(15: 1 \mathrm{v}: \mathrm{v})$ entwickelt. Das Chromatogramm 1036-a wurde mit Phosphor-molybdänsäure-Reagens nach P. H. List - und das Chromatogramm 1036-b mit Dragendorff-Reagens, gefärbt

Verbindung entstehen aus dem Spartein noch andere Abkömmlinge, von denen mengenmässig den grössten Anteil derjenige innehält, der auf dem Chromatogramm zwischen Lupanin und dem ,Alkaloid“ $\mathrm{n}_{6}$ erscheint. Die Abbildung 11 stellt das Spartein und seine Abbauprodukte dar.

Weitere Versuche zeigten, dass das Sparteinhydrochlorid fast völlig gegen die einwirkenden Reagentien widerstandsfähig ist.

In der Alkaloidchemie trifft es sich sehr oft, dass die Salze verschiedener synthetischer Basen genug Widerstandskraft besitzen, so, dass ihre physikalischen Daten festgestellt werden können, während die entsprechenden freien Basen unmittelbar nach ihrem Entstehen einer Zersetzung unterliegen. Obwohl das Spartein eine bekannte, durch Synthese bewiesene Konstitution besitzt, ist es jedoch weiterhin ein Alkaloid, welches 
die Chemiker sehr interessiert. Die räumliche Anordnung der Wasserstoffatome bei Kohlenstoffatom 6 und 11 ist unsicher (Abb. 1). Die langdauernden Arbeiten von L. M a rion und N. J. L e o n a d (1951) und besonders von F. Galinovsky und Mitarb. (1954-55) führen zu dem Schluss, dass das Spartein aus trans- und cis-Norlupinan-Ringen, die in Position 1,3 verbunden sind, aufgebaut ist. Die Anwesenheit, der überhaupt nicht haltbaren Anordnung cis-Norlupinan in dem Sparteinmolekül, begünstigt die zu einer Verminderung des Energiezustandes des Moleküls führenden Umsetzungsprozesse. In letzter Zeit gelang es F. G a linovsky (1954-55) durch Einwirken mit Aluminiumchlorid und darauffolgender Unterdruckdestillation das $\alpha$-Isospartein in Form von Kristallen zu erhalten. Es wird angenommen, dass diese Verbindung aus zwei trans-Norlupinan-Anordnungen besteht. Im Lichte der angeführten Arbeit von F. G a lin ovsky erschien es eigentümlich, dass bisher die Zerfallsprodukte der Sparteinbase und ihrer Lösungen noch nicht genau untersucht wurden, obwohl dieser Zerfall ohne äussere Einwirkung oft beobachtet wurde. Wir nehmen an, dass systematische chromatographische Untersuchungen viel zur Identifizierung der Zerfallprodukte der Sparteinbase beitragen könnten, was unserer Ansicht nach von grosser Bedeutung für Untersuchungen über die Biogenese der Lupinenalkaloide sein würde. Diesen Untersuchungen wird gegenwärtig grosse Aufmerksamkeit gewidmet.

Im Laufe der zuletzt beschriebenen Untersuchungen unterlag auch Lupinin und das "Alkaloid“ $\mathrm{n}_{6}$ bestimmten Umsetzungen. Die Lupininbase geht unter Einwirkung von Wasserstoffperoxyd in eine Verbindung über, die ein wenig grössere $R_{f}$-Werte wie das ,Alkaloid“ $n_{6}$ aufweist; ebenfalls unter mehrmaligem Einwirken von Chloroform erfährt sie eine Zersetzung.

In diesem Falle bildet sich eine Verbindung, die chromatographisch dem Spartein ähnlich ist. Das „Alkaloid“ $\mathrm{n}_{6}$ zersetzt sich unter Einfluss von konz. Salzsäure und ebenfalls unter Einwirkung von Wasserstoffperoxyd. In beiden Fällen erhielten wir auf Chromatogrammen einen Ring mit grösserem $\boldsymbol{R}_{\mathrm{f}}$-Wert als den des „Alkaloids“ $\mathrm{n}_{6}$.

Die zuletzt angeführten Untersuchungen sehen wir nur als Einführungsarbeiten an. Sie erlaubten uns die Widerstandsfähigkeit einiger Lupinenalkaloide gegen verschiedene Reagentien bei Zimmertemperatur näher kennenzulernen. Den Untersuchungskreis wollen wir in Zukunft durch Einführung verschiedener Temperaturen und Mischungen von natürlichen und synthetischen Alkaloiden weiter ausdehnen. 
EINIGE EINZELHEITEN UUBER DAS MATERIAL UND DİE VERSUCHSTECHNIK

\section{a. Das biologische Material und Reagentien}

Die von uns untersuchten Lupinensamen stammten aus:

1. Schmalblättrige Lupine, - rosa, bitter — aus der Station für Samenzucht Udycz, Station Podkowa Leśna bei Warszawa, Ernte 1951.

2. Schmalblättrige Lupine, - blau, bitter - aus der Station für Samenzucht des IHAR in Miników, Ernte 1953.

3. Weisse Lupine, - bitter - aus der Station für Samenzucht des IHAR in Przebendowo, Ernte 1953.

4. Gelbe Lupine, - bitter - aus der Station für Samenzucht des IHAR in Miników, Ernte 1953.

5. Mehrjährige Lupine - aus dem Institut für Pflanzensamenkunde der WSR in Poznań, Ernte 1952.

Die angewandten Reagentien waren chemisch rein, wobei alle Lösungsmittel, ohne Rücksicht auf die Qualität, noch mindestens einmal destilliert wurden. Die von uns angewandten Vergleichssubstanzen: Lupinin, Lupanin, Hydroxylupanin und Spartein stammten ausschliesslich von der Firma Merck Darmstadt.

\section{B. DAS CHROMATOGRAPHISCHE VERFAHREN}

\section{Filtrierpapier und Rundfilter}

Anfangs gebrauchten wir zur chromatographischen Analyse quantitative Rundfilter von der Firma J. H. Munktell $\mathrm{nr}$ Ok und $\mathrm{Ob}$, nachher führten wir die Analyse ausschliesslich auf Filtrierpapier Whatman nr. 1 aus. Aus einem Bogen Filtrierpapier schnitten wir zwanzig Stück Rundfilter $\varnothing 11 \mathrm{~cm}$ aus. Vor Ausschneiden der Rundfilter vermerken wir den Durchmesser parallel zur Fasserrichtung. Nachher zeichnen wir um den Mittelpunkt einen Kreis $\varnothing 14 \mathrm{~mm}$ und schneiden dann mit einer Rasierklinge parallel zur Faserrichtung eine "Zunge“ (4×40 mm, oder $2 \times 40 \mathrm{~mm}$ ) aus. Den Umfang des kleinen Kreises ( in soviel gleiche Teile, wie viel Substanzen auf dem Chromatogramm analysiert werden sollen. Den Kreisabschnitt auf den die „Zunge“ fällt, lassen wir aus. Wenn das Rundfilter vorher impregniert werden soll, wenden wir ein normalisiertes Verfahren an, indem wir es auf $10 \mathrm{~min}$ in die Impregnationslösung legen. Nach dem Herausnehmen halten wir das Rundfilter in senkrechter Lage 3 Min., bringen es dann auf reines Filtrierpapier, wo es $10 \mathrm{Min}$. verbleibt, um es endlich auf eine Glasplatte zu legen. Das Rundfilter trocknet auf der Glasplatte so lange, bis sein Feuchtigkeitsgrad mit dem der Luft in Gleichgewicht kommt. 


\section{Das Auftragen der Lösung auf das Rundfilter}

In den bisherigen Versuchen, die die qualitative Analyse zum Ziele hatten, wandten wir zum Auftragen der Lösung eine selbst hergestellte „Oese“ aus Platindraht an. Diese „Oese“ besass meistens einen Durchmesser von $0,6-1,0 \mathrm{~mm}$, und ein Volumen von $0,4-1,0 \mu \mathrm{l}$ (das Volumen wurde gegenüber Wasser bestimmt). Eine wässrige Lösung - mit Hilfe einer solchen „Oese“ auf Filtrierpapier gebracht - bildet einen runden Fleck von $\varnothing 2-4 \mathrm{~mm}$. Die zu untersuchende Alkaloidlösung engen wir vor dem Aufbringen zu einer möglichst hohen Konzentration ein, so, dass eine „Oese“ Lösung genügend viel Substanz enthält. Nach dem Aufbringen der Lösung, tragen wir auf den Startpunkt noch eine „Oese“ 0,5-nSalzsäure auf.

3. Die zum Entwickeln angewandten Lösungsmittelsysteme und ihre Vorbereitung

In unseren Arbeiten wenden wir hauptsächlich das Lösungsmittelsystem ,"wassergesättigter n-Butanol“ an, dessen Vorbereitung und Regenerierung keine Schwierigkeiten bieten.

Das zweite von uns oft angewandte Lösungsmittelsystem ist eine Mischung von 4-m-Ammoniumsulfatlösung und Aethanol (15:1) (v:v). Durch Ergänzung des Lösungsmittelsystems von A. Resplondy (1955) mit Aethanol erzielt man ein besseres Aussehen des Chromatogrammes. Das mit 4-m-Ammoniumsulfatlösung entwickelte Chromatogramm weist zwar eine deutliche Trennung des Lupinins vom Lupanin auf, aber die Alkaloidringe stellen ein welliges Bild (Girlandenmuster) dar, was eine Ermittlung der $R_{\mathrm{f}}$-Werte völlig unmöglich macht. Der von uns angewandte Aethanolzusatz beseitigt nicht nur völlig das Auftreten von Girlandenmustern, sondern erzeugt auch ein markanteres Erscheinen der Ringumrisse. Das Lösungsmittelsystem stellen wir her, indem wir zu einem kleinen Teil 4-m-Ammoniumsulfatlösung, unter dauerndem Mischen, tropfenweise den Aethanol hinzugeben, bis die anfangs auftretende Emulsionsbildung verschwindet. Anstatt der 4-m-Ammoniumsulfatlösung wenden wir oft gesättigte Lösungen dieser Verbindung an, die je nach der Temperatur eine höhere oder niedrigere Konzentration als $4 \mathrm{Mol} / \mathrm{l}$ besitzen, was jedoch keine besondere Bedeutung hat.

\section{Das Konditionieren*}

Anfangs führten wir das Konditionieren und Entwickeln des Chromatogramms in Exsikkatoren aus M. Wiewiórowski u. M. D. B r a t e k (1956), sind jedoch nach den Vorschlägen von J. S e if ers und

* Unter „Konditionieren“ verstehen wir eine Sättigung des Rundfilters mit Dämpfen das Lösungsmittelsystems welches zum Entwickeln des Chromatogrammes dient. 
J. O reskes (1953) zum Entwickeln in Kristallisierschalen $(\varnothing 10 \mathrm{~cm}$, $\mathrm{h}=4 \mathrm{~cm}$ ) übergegangen, die wir mit Deckschalen von Petrischalen abschliessen. In die Kristallisierschalen bringen wir kleinere $(\varnothing 4 \mathrm{~cm}$, $\mathrm{h}=3 \mathrm{~cm}$ ) die mit dem Lösungsmittelsystem gefüllt sind — wobei wir darauf achten, dass der Abstand zwischen Flüssigkeitsoberfläche und Rundfilter immer gleich ist. In den meisten Fällen betrug der Abstand zwischen Lösungsmittel und Rundfilter $14 \mathrm{~mm}$. Die Chromatogramme werden vor dem Entwickeln immer in Petrischalen konditioniert (Ø $10 \mathrm{~cm}$ ). Die Unterschale wurde mit $10 \mathrm{ml}$ Lösungsmittelsystem beschickt und das Rundfilter mit augebrachted Substanz aufgelegt, wobei die „Zunge“ etwas nach oben abgebogen wurde. Nachher wurde die Deckschale aufgesetzt und das Rundfilter, welches einen grösseren Durchmesser als die Schale hat, eingedrückt und zusätzlich beschwert:

Nach 1 Stunde wird die Deckschale mitsamt Rundfilter abgehoben, die ,Zunge“ mit einer Pinzette in senkrechte Lage gebracht, worauf das Rundfilter mit Deckschale auf die Kristallisierschale gesetzt werden, um je nach Art des Lösungsmittelgemisches, einmalig oder mehrmalig zu entwickeln. Wenn mit wassergesättigtem n-Butanol als Lösungsmittelsystem gearbeitet wird, entwickeln wir zweimal. Vor wiederholtem Entwickeln des Chromatogramms wird nochmals ,,konditioniert“. Konditionieren und Entwickeln führen wir in einem möglichst kühlen Raum durch, in dem die Temperaturschwankungen möglichst gering sind.

5. Die Entwicklungsreagenzien und das Sichtbarmachen der auf dem Chromatogramm verteilten Substanzen

Die Alkaloidflecke auf dem entwickelten Chromatogramm machen wir meistens mit dem von R. Munier und Mitarb. modifizierten D ragendorff - Reagens sichtbar.

Die Vorbereitung des Reagens:

Die Grundlösung erhält man durch Vereinigung der Lösungen A und $B$, die aus folgenden Bestandteilen bestehen:

Lösung. A: $850 \mathrm{mg}$ Wismut-III-nitrat, bạs. (,Bismutum subnitricum“), $40 \mathrm{ml}$ dest. Wasser,

$10 \mathrm{ml}$ Eisessig,

Lösung B: 8 g Kaliumjodid, $20 \mathrm{ml}$ dest. Wasser.

Die Grundlösung, in Braunglasflaschen aufbewahrt, ist ebenso wie das verdünnte Reagens monatelang haltbar. Zum Färben der Chromatogramme muss die Grundlösung mit Eisessig und dest. Wasser verdünnt werden (meistens mischt man die erwähnten drei Teile des Reagens in folgendem Verhältnis: $10: 20: 100 . \mathrm{v}: \mathrm{v}: \mathrm{v}$ ). 
Das Chromatogramm wird, nach Trocknen an der Luft, in das Reagens getaucht und nach 15 Min. herausgenommen und auf reines Filtrierpapier gebracht, wo es wiederum an der Luft trocknet. Eine Unzulänglichkeit des D r a g e n d o r f $f$-Reagens ist die teilweise Abscheidung der farbigen Alkaloidverbindungen aus dem Chromatogramm, was sich in einer leichten Trübung des Reagens bemerkbar macht. Eine solche Störung tritt jedoch nur bei grosser Alkaloidkonzentration auf. Das von uns angewandte $15 \mathrm{Min}$. dauernde Baden des Chromatogramms in der Reagenslösung bewirkt eine Gleichgewichtsverschiebung des anfangs labilen Systems: farbiger Alkaloidkomplex - Reagenslösung, zu Gunsten des auf dem Filtrierpapier haftenden Alkaloidkomplexes. Auf diese Weise konnten wir das störende Ausscheiden der Alkaloide aus dem Chromatogramm und das Auftreten verwischter Ringkonturen beseitigen. Falls auf dem Chromatogramm Lupinin anwesend sein sollte, darf man nicht zu lange baden, da es mit dem Dragendorff -Reagens eine leicht lösliche Verbindung bildet, die völlig in Lösung übergehen kann.

Das von H. Thies und W. F. Reuther (1955) modifizierte Dra ge n d o r ff-Reagens weist den Lupinenalkaloiden gegenüber keine besonderen Eigenschaften auf.

Viel besser ist das für quantitative Zwecke anwendbare Reagens mit Phosphormolybdänsäure nach P. H. List (1954). Die Vorbereitung sowie die Färbung der Chromatogramme verläuft wie folgt: 1 g Phosphormolybdänsäure löst man in einer Mischung von konz. Salpetersäure Wasser (2: $98 \mathrm{v}: \mathrm{v})$ auf. Das Chromatogramm wird durch die Lösung gezogen und dann $10 \mathrm{Min}$. in fliessendem Wasser gewaschen. Nachher trocknet man auf reinem Filtrierpapier und zieht dann das Chromatogramm duch eine Zinn-(11) - chloridlösung (1 g Zinn-(II)- chlorid, $100 \mathrm{ml} \mathrm{3,8 \%}$ Salzsäure), und trocknet wieder auf Filtrierpapier. Zu quantitativen Zwecken muss die Zeit, während der das Chromatogramm mit der Phosphormolybdänsäure und der Zinn-(II)- chloridlösung in Berührung ist, unbedingt normalisiert werden, weil auch in diesem Falle ein Uebergehen der farbigen Alkaloidkomplexe in die Reagenslösung stattfindet.

Das Dragendorff-Reagens und das Reagens mit Phosphormolybdänsäure besitzen ähnliche Empfindlichkeit gegenüber den Lupinenalkaloiden. Das Thalliumacetat-Reagens nad R. M u n i e r und Mitarb. (1949-52) besitzt eine ähnliche Empfindlichkeit, es ist jedoch von uns nur selten gebraucht worden, da es farbige Flecken von geringerem Kontrast gegen den Filtrierpapieruntergrund bildet. Die Färbungstechnik mit diesem Reagens ist ähnlich wie die im Falle von DragendorffReagens. 
C. DIE ALKALOIDEXTRAKTION FUR DIE QUALITATIVE, CHROMATOGRAPHISChE ANALYSE

$\mathrm{Zu} 2$ g gemahlener Lupinensamen geben wir in einem Mörser $3 \mathrm{ml}$ konz. Ammoniaklösung und zerreiben, mit kleinen Pausen. Nachher geben wir unter ständigem Rühren 3 g Kieselgur oder Kalziumsilicat hinzu (das Kalziumsilicat und Kieselgur müssen vor Gebrauch $3 \mathrm{~h}$ hei einer Temperatur von $250^{\circ} \mathrm{C}$ getrocknet werden).

Die trocken aussehende Masse bringen wir in eine Röhre $(12 \times 250 \mathrm{~mm})$, in die vorher ein Watteknäuel geschoben wurde. Das Anfüllen der Chromatographiesäule wird möglichst normalisiert ausgeführt so, dass die Ausflussgeschwindigkeit der Extrakte $10-15 \mathrm{ml} / \mathrm{h}$ beträgt. Als Lösungsmittel zum Extrahieren der Alkaloide aus der Säule wenden wir frisch gereinigten Chloroform an. (Chloroform reinigen wir nach der üblichen Methode). Die Extraktion führen wir meistens auf diese Weise aus, dass wir in die Säule $10 \mathrm{ml}$ Chloroform bringen, die Säule oben abschliessen und über Nacht stehen lassen. Am nächsten Morgen fangen wir mit der eigentlichen Extraktion an, indem wir durch die Säule $40 \mathrm{ml}$ Chloroform durchlaufen lassen. Den abtropfenden Alkaloidextrakt sammeln wir in einem Jenaer Acetylierungskolben (100 ml.). Den Chloroformextrakt destillieren wir ab, geben $\mathrm{zu}$ dem fast trockenen Rückstand ungef. 0,5 ml Methanol und erhitzen einige Minuten auf dem Wasserdampfbad, um den Chloroform völlig auszutreiben. Nachher wird $1 \mathrm{ml}$ 0,1 n-Salzsäure hinzugegeben, noch ein wenig erwärmt, und nach $\mathrm{Ab}$ kühlen durch Filtrierpapier in ein Reagensglas $(12 \times 40 \mathrm{~mm})$ abfiltriert. Der Acetylierungskolben wird zweimal mit Wasser ausgespült $(2 \times 0,75 \mathrm{ml})$. Nach jedem Spülen wird der Kolben leicht erhitzt, wieder gekühlt und das Spülwasser in das gleiche Reagensglas abfiltriert. Die nach diesem Verfahren erhaltene Alkaloidlösung ist völlig klar und beinahe farblos.

Die saure Lösung dampfen wir in einem Vakuum-Exsikkator über Phosphorpentoxyd und Natriumhydroxyd völlig ein und den erhaltenen Rückstand tragen wir, nach Aufnehmen in einem Tropfen Wasser, mit der Platindrahtöse auf das Rundfilter auf. Das so hergestellte Chromatogramm kann nun „konditioniert" und entwickelt werden.

\section{ABSCHLUSS}

Die angeführten Ergebnisse, welche die von uns in den letzten vier Jahren ausgeführten Arbeiten über die qualitative Alkaloidzusammensetzung in Lupinensamen betreffen, erlaubten uns nicht das erstrebte Ziel zu erreichen.

Die gleichzeitige Ausführung vieler und oft sehr verschiedener Untersuchungen erschwerten es diese ausführlich zu bearbeiten. 
Einzelne Teile noch nicht beendeter Arbeiten, sowie geplante Untersuchungen wurden von uns absichtlich angeführt. Wir wollen nämlich dadurch andere Forscher anregen sich mit der Analyse und der Physiologie der Lupinenalkaloide zu befassen. Diese Meinung drücken wir deshalb aus, weil wir befürchten, dass wir allein mit den Schwierigkeiten, deren Anzahl bestimmt grösser ist als wir besher annahmen, nicht fertig werden.

An dieser Stelle wollen wir unseren herzlichen Dank aussprechen: an Prof. Dr. J. Suszko für die Aufstellung des Untersuchungsthemas und für zahlreiche Anregungen, an Prof. Dr. S. B a r b a cki für die wertvollen Anleitungen und für die Beschaffung des Untersuchungsmaterials, sowie Doc. Dr. B. Borkowski für die Überlassung von Lupanin-, Lupinin- und Cytisin-Präparaten.

Die Arbeit wurde in den Jahren 1951-1955 im Institut für Allgemeine Chemie der Oekonomischen Hochschule in Poznań, und im Institut für Pflanzenzucht, der Polnischen Akademie der Wissenschaften, in Poznań, ausgeführt.

\section{ZUSAMMENFASSUNG}

Als Ziel der vorliegenden Arbeit wurde eine genaue Bestimmung der qualitativen Alkaloidzusammensetzung von Lupinensamen erstrebt und mit Hilfe von papierchromatographischen Methoden zu erreichen versucht. Die angewandte chromatographische Technik, einige Lösungsmittelgemische und Entwicklungsreagentien wurden beschrieben. Besondere Aufmerksamkeit wurde auf die unvorhergesehenen Schwierigkeiten gelegt, die bei der chromatographischen Trennung des Lupinins vom Lupanin auftraten. Es wurde ebenfalls das merkwürdige Verhalten des Sparteins besprochen, das sich in manchen Fällen auf einem Chromatogramm an zwei verschiedenen Stellen ablagert. Mit Hilfe eigener chromatographischer Arbeitsmethoden wurden verschiedene übliche Extraktionsverfahren für Lupinensamenalkaloide untersucht und kritisch bewertet.. Unter Berücksichtigung der Analysenergebnisse wurde ein neues Extraktionsverfahren für die Gesamtalkaloide sowie ein Verfahren zur fraktionierten Auswaschung einzelner Alkaloide aus Samen der weissen und schmalblättrigen Lupine beschrieben. Nach Anwendung einer einheitlichen Extraktion und normalisierten chromatographischen Technik konnte die qualitative Alkaloidzusammensetzung der weissen, schmalblättrigen, mehrjährigen und gelben Lupine bestimmt werden. Es wurde festgestellt, dass die Alkaloidzusammensetzung der genannten Lupinenarten komplizierter ist, als bisher angenommen wurde.

In weisser, schmalblättriger und mehrjähriger Lupine wurden ausser Lupanin und Hydroxylupanin noch fünf weitere organische Basen festgestellt, die mit den üblichen Alkaloidreagentien positiv reagieren.

Die neuentdeokten Alkaloide wurden , $\mathrm{n}_{1}$ “, ,, $\mathrm{n}_{2}$ ", ,, $\mathrm{n}_{3}$ ", , , $\mathrm{n}^{4 / 5}$ " und , , $\mathrm{n}_{6}$ " benannt. Ausserdem wurde die Anwesenheit des Sparteins in der mehrjährigen und des Lupinins in der schmalblättrigen Lupine nachgewiesen. 
Die $R_{f}$-Werte von neun Lupinenalkaloiden wurden für zwei Lösungsmittelgemische (1. wassergesättigter n-Butanol, 2. 4m-Amoniumsulfatlösung und Aethanol, $15: 1 \mathrm{v}: \mathrm{v})$ festgelegt. Während der Identifikationsproben der neuentdeckten "Alkaloide“ wurden Schwierigkeiten festgestellt, die bei der präparativen Abscheidung des wenig beständigen .,Alkaloids $\mathrm{n}_{3}$ " auftraten. Ausserdem wurden einige Eigenschaften des "Alkaloids $\mathrm{n}_{6}$ " beschrieben, das in Milligrammengen als chemisch und chromatographich reine Substanz erhalten werden konnte. Im Zusammenhang mit der Unbeständigkeit des ,Alkaloids $n_{3}$ “ wurden Versuche zur Bestimmung der Haltbarkeit und der Widerstandsfähigkeit gegen Extraktionsmittel und Faktoren, die während der chromatographischen Analyse einwirken, für die einzelnen Alkaloide unternommen. Einführungsarbeiten auf diesem Gebiete zeigten, dass Alkaloidsalze weniger abbau- und umsatzfähig sind als die freien Alkaloidbasen. Besonders abbau- und umsatzfähig ist das Spartein, wobei festgestelit wurde, dass sein Hauptabbauprodukt auf Chromatogrammen die gleiche Stelle einnimmt wie das ,Alkaloid $\mathrm{n}^{4} / 5$ ". Auch Lupinin und das "Alkaloid $\mathrm{n}_{6}$ " unterliegen deutlich nachweissbaren Umsetzungen; während Lupanin und Hydroxylupanin, sogar nach längerer Einwirkung von $3 \% \quad \mathrm{H}_{2} \mathrm{O}_{2}$, keine deutlichen Änderungen aufweisen.

Weitere Untersuchungen sind im Gange.

(Eingegangen 17.5.1956)

Achmatowicz O. i Rodewald W., 1955, Roczniki Chemii 29: 509.

B a rba cki S., 1952, Łubin, P. W. R. i L., Warszawa.

Bellet P., 1950, Ann. pharm. franc. 8: 551.

Burden E. H. W. J., Grindley D. N., M ans our R., 1954, J. Pharm. Pharmacol. 6: 461 .

Burma A. P., 1954, Naturwissenschaften 41: 19.

Clemo G. R., Leith G. C., 1928, J. Chem. Soc. 18: 11.

Galinovsky F., 1952, Fortschritte der Chemie organischer Naturstoffe 8: 245.

Galinovsky F., Ka inz G., 1951, Monh. Chem. 82: 927.

Galinovsky F., Knoth P., 1955, Naturwissenschaften 41: 454 (54); Monh.

Chem. 86: 1014.

Giri K. V., R a o N. A. N., 1952, Nature 169: 923.

Graf E., 1952, Dtsch. Apoth. Ztg. 91: 797.

Hackeman R. H., Lazarus IM., 1955, Biochim. et Biophys. Acta 17: 147.

Henry T. A., 1950, The Plant Alkaloids, London.

Lis t P. H., 1954, Naturwissenschaften 41: 454.

Leon ard N. J., 1953, The Alkaloids Chemistry and Physiology 3: 119, New York. Marion L., Le on ard N. J., 1951, Canad. J. Chem. 29: 355.

Munier R., Ma cheboeuf M., 1949, Bull. Soc. chim. biol. 31: 1144.

Munier R., Ma cheboeuf M., 1950, Bull. Soc. chim. biol. 32: 192.

Munier R., Ma cheboeuf M., 1951, Bull. Soc. chim. biol. 33: 846.

Munier R., Macheboeuf M., Cherrier N., 1951, Bull. Soc. chim. biol 33: 1919.

Munier R., Macheboeuf M., Cherrier N., 1952, Bull. Soc. chim. biol. 34: 204. 
Allouf R., Munier R., 1952, Bull. Soc. chim. biol. 34: 196.

Reifer I., Możejko M., 1954, Acta Biochimica Polonica 1: 197.

Rink M., Grabowski K., 1955, Naturwissenschaften 42: 343 .

Resplondy A., Sa unie Ch., 1955, C. R. Acad. Sci. 241: 65, Paris.

Rutter L., 1948, Nature 161: 435.

Seifer A., Oreskes J., 1953, Anal. Chem. 25: 1539.

Thies H., Reuther F. W., 1954, Naturw:ssenschaften 41: 230.

Thies H., Reuther F. W., 1955, Naturwissenschaften 42: 462.

Wierzchowski Z., Obuchowska L., 1952, Rocznjki Nauk Rolniczych 61: 159.

Wiew í́rowski M., Bratek M. D., 1956, Bull. de Acad. Polon. des Sci. 4: $(1,3)$.

Wiew iórowski MI., Arbeit nicht veröffentlicht. 